\title{
Aula invertida: Una propuesta en la enseñanza de la histología veterinaria
}

\section{Flipped classroom: A proposal in the teaching of veterinary histology}

\section{Aula invertida en histología veterinaria}

\author{
José Yaguana Jiménez ${ }^{(1)}$ \\ Laura de Jesús Peña Merino ${ }^{(2)}$ \\ Edison Riveliño Ramón Curay, ${ }^{(3)}$
}

(1) Facultad de Medicina Veterinaria y Zootecnia. Docente de la Universidad Nacional de Loja, Ecuador. email:pepeysesa@yahoo.com

(2) Facultad de Medicina Veterinaria y Zootecnia. Docente de la Universidad Nacional de Loja, Ecuador. email:laura.pena@unl.edu.ec

(3) Facultad de Medicina Veterinaria y Zootecnia. Docente de la Universidad Estatal de Bolívar. email:edison_rivelino@yahoo.com

Contacto:pepeysesa@yahoo.com

Recibido: 13-04-2020 Aprobado: 23-05-2020

\section{Resumen}

En la actualidad en la mayoría de las universidades, están optando por implementar modelos pedagógicos que permitan la innovación educativa, uno de estos modelos es el del aula invertida, que ha ido ganando popularidad en los docentes $\mathrm{y}$ estudiantes.El presente artículo tiene como objetivo implementar el modelo del Aula Invertida en la enseñanza de la histología veterinaria en la Carrera de Medicina Veterinaria y Zootecnia en la Universidad Nacional de Loja, Ecuador.El tipo de investigación fue exploratorio-descriptivo, con un diseño no experimental. Los sujetos de estudio fueron 30 estudiantes, y se empleó como herramienta la encuesta. Los principales resultados resaltan la disposición de los educandos en utilizar el modelo del aula invertida, se fomenta el trabajo autónomo y se eleva la motivación y la colaboración de los estudiantes.Se revela una alta audiencia en la consulta de videos de histología veterinaria dentro y fuera del aula tradicional; empleando las redes sociales como YouTube. Se concluye que en el estudio realizado se pudo conocer que los estudiantes y docentes tienen conocimiento del modelo del aula invertida y manifiestan que les gustaría implementarlo en la asignatura de histología veterinaria, es evidente que esto puede elevar la motivación y fortalecer la innovación pedagógica. Además, este modelo promueve el trabajo autónomo y la colaboración de los educandos en el uso de los vídeos educativos dentro y fuera del aula tradicional; empleando las redes sociales como YouTube.

Palabras clave:Aula invertida; histología veterinaria; enseñanza universitaria.

\begin{abstract}
Currently in most universities, they are choosing to implement pedagogical models that allow educational innovation, one of these models is that of the inverted classroom, which has been gaining popularity among teachers and students. This article aims to implement the model of the Inverted Classroom in the teaching of veterinary histology in the Veterinary Medicine and Zootechnics Degree at the National University of Loja, Ecuador. The type of research was exploratorydescriptive, with a non-experimental design. The study subjects were 30 students, and the survey was used as a tool. The main results highlight the willingness of the students to use the inverted classroom model, autonomous work is encouraged, and the motivation and collaboration of the students are increased. A high audience is revealed in the consultation of veterinary histology videos inside and outside of the traditional classroom; using social networks like YouTube. It is concluded that in the study carried out it was known that students and teachers have knowledge of the inverted classroom model and state that they would like to implement it in the subject of veterinary histology, it is evident that this can increase motivation and strengthen pedagogical innovation. In addition, this model promotes the autonomous work and collaboration of students in the use of educational videos inside and outside the traditional classroom; using social networks like YouTube.
\end{abstract}

Keywords: Flipped classroom;veterinary histology; university teaching. 


\section{Introducción}

En los últimos años los avances tecnológicos han propiciado cambios importantes en los escenarios educativos universitarios y por otra parte se aprecia una buena disposición de los estudiantes en utilizar los recursos digitales en las aulas. En este sentido uno de los retos a los que se enfrenta las universidades en Ecuador, es la implementación de nuevos modelos que permitan potenciar la innovación pedagógica y una mayor flexibilidad y protagonismo por parte de los educandos en las aulas, una de estas metodologías es el aula invertida o flipped classroom.

Según Berenguer (2016), el aula volteada ha ganado una gran popularidad en los docentes universitarios, el autor asevera que el objetivo de este modelo es que el alumno tome un rol más activo en su proceso de aprendizaje, se caracteriza por invertir los momentos y roles de la enseñanza tradicional, donde la clase impartida por el profesor, pueda ser atendida en horas extra-clase por el estudiante mediante las herramientas de vídeos; los estudiantes adquieren una responsabilidad de su aprendizaje, adecuándose a su propio ritmo, su espacio y tiempo, el maestro se convierte en una guía en vez del exponente de una clase.

Es importante significar los aportes de Bergmann \& Sams (2012), se encargaron de consolidar el término de flipped classroom también lo popularizaron, los autores afirman que se trata de un enfoque pedagógico en el que la instrucción directa se mueve desde el espacio de aprendizaje colectivo hacia el espacio de aprendizaje individual, y el espacio resultante se transforma en un ambiente de aprendizajedinámico e interactivo en el que el docente guía a los alumnos a medida que se aplican los conceptos y puede participar creativamente en la materia.

Bergmann \& Sams (2012), afirman que para implementar el modelo, el primer paso consiste en realizar una sesión presencial, con el propósito de lograr motivar a los estudiantes a revisar el material multimedia preparado, es recomendable que sea un vídeo corto, de fácil el acceso para su visualización. Tambiénrecomiendan suministrar material impreso o cuestionarios donde se evidencie la toma de apuntes de la visualización de las presentaciones.

Cabe mencionar que en el contexto de la Educación Superior en Ecuador, el modelo no está muy extendido en la Carrera de Medicina Veterinaria y Zootecnia, pero en los últimos años se ha notificado un gran avance en lo relativo a las tecnologías y las herramientas de la web 2.0, lo que ha propiciado un aumento de iniciativas en varias instituciones que han optado por implementarlo, alcanzando resultados favorables y una gran aceptación por parte de los estudiantes (Rivera \& García 2018).

El presente trabajo tiene como objetivo valorarla implementacióndel modelo del Aula Invertida en la enseñanza de la histología veterinaria en la Carrera de Medicina Veterinaria y Zootecnia en la Universidad Nacional de Loja, Ecuador.

\section{Definiciones del Aula Invertida}

Kachka (2012), afirma que es un modelo de enseñanza que cambia la forma tradicional de realizar una clase, cambia la forma de empleo del tiempo dentro y fuera del aula, otorgando a los estudiantes la responsabilidad de su aprendizaje, adecuándose a su propio ritmo, su espacio y tiempo. El docente se convierte en una guía en vez del exponente de una clase.

Para Tourón \& Santiago (2015), es un modelo didáctico en el cual los estudiantes aprenden nuevo contenido a través de videos-tutoriales en línea, habitualmente en casa; y lo que antes solían ser los "deberes" (tareas asignadas), se realizan ahora en el aula con el profesor ofreciendo orientación más personalizada e interacción con los estudiantes.

Para otros investigadores como Monteagudo, Gómez \& Miralles (2017), aseveranque se trata deun modelo pedagógico que se basa en trasladar determinadas actividades del proceso de aprendizaje, fuera del entorno del aula y utiliza el tiempo de clase, y se apoyaen la experiencia del profesorado, para facilitar y potenciar otros procesos de adquisición y práctica de conocimientos dentro del aula.

Es evidente que el modelo del aula invertida, propicia un mayor protagonismo y responsabilidad en su formación por parte de los estudiantes, el docente asume un nuevo rol y se convierte en un facilitador del aprendizaje. Este modelo se apoya en el uso de tecnología multimedia (video conferencias, presentaciones).Otro aspecto a destacar es que el aprendizaje tiene lugar en cualquier lugar y en cualquier momento y se lleva a cabo a partir de las experiencias de los estudiantes, de la conversación con sus compañeros y docentes.

\section{Material y métodos}

La investigación se llevo a cabo en la Facultad de Medicina Veterinaria y Zootecnia en la Universidad Nacional de Loja, Ecuador. La muestra estuvo conformada por 30 estudiantes que 
se encontraban cursando el ciclo de la asignatura de histología veterinaria en el año 2019.Así como el profesor encargado de impartir esta asignatura, tuvo la iniciativa de proponer la implementación del modelo del aula Invertida o flipped classroom.

El estudio estuvo enfocado en conocer el criterio de los estudiantes y el profesor sobre el empleo del modelo del Aula Invertida.La investigación se desarrolló bajo un enfoque cuantitativo de tipo exploratorio-descriptivo.

Se realizó una encuesta, compuesta por 9 preguntas, 3 de ellas se diseñaron según la escala de Likert, con una sola opción de cinco posibles respuestas: siempre, casi siempre, a veces, casi nunca, nunca. La encuesta se les realizó a los estudiantes en el aula tradicional a todos los sujetos que formaron parte de la investigación.

\section{Resultados}

Una vez aplicada la encuesta a la muestra conformada por 30 estudiantes del curso

de Histología Veterinaria en la Facultad de Medicina Veterinaria y Zootecnia de la Universidad Nacional de Loja, Ecuador. Los resultados se tabularon y representaron gráficamente. En la figura 1, se muestran los obtenidos en las primeras seis preguntas y en la figura 2, las tres restantes relacionadas con las acciones que realiza el docente sobre el uso y promoción de los recursos educativos.
¿Conoces el modelo de Aula Invertida o Flipped Classroom?

¿Te gustaría implementar el Modelo del Aula Invertida, el proceso de aprendizaje?

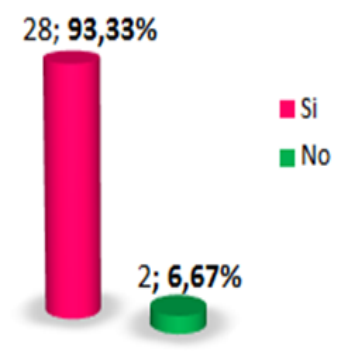

¿Compartes conocimientos con tus compañeros relacionado con el uso de las TIC y los vídeos relacionados con la asignatura?

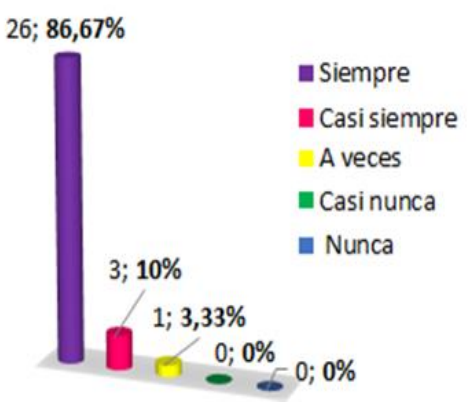
con el propósito de fortalecer

¿Consultas vídeos de histología veterinaria fuera del aula tradicional?

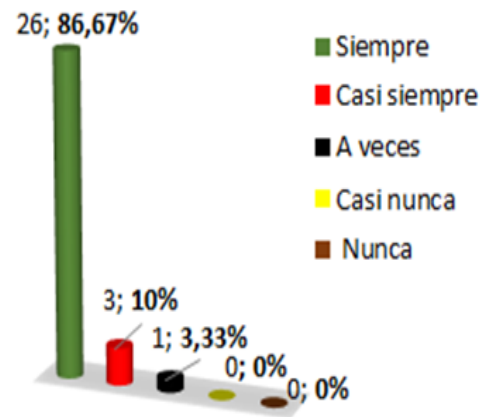

¿Consideras que el modelo del Aula Invertida, fomenta en ti el trabajo autónomo?

¿Consideras que el modelo del Aula Invertida eleva tu motivación más que el de las clases tradicionales?

Figura 1. Resultados del procesamiento de las seis primeras preguntas de la encuesta.

Con la intensión de conocer si los estudiantes saben lo que es el modelo de aula invertida y si les gustaría implementarlo, se formularon las dos primeras preguntas. Como se puede apreciar en las primeras dos gráficas de la figura 1, con igual porcentaje $(93,33 \%)$ los educandos opinaron de forma afirmativa estas interrogantes. Estos criterios resultan favorables si se aplicara este modelo, dado que la disposición de los implicados en un elemento fundamental en el logro de buenos resultados.

Dada las ventajas que se reconoce que tienen los videos para lograr que los estudiantes comprendan los contenidos sin la presencia física del profesor, se considera satisfactorio el hecho de que el 86,67 $\%$ de los encuestados como se muestra en la tercera gráfica de la figura 1, opinaran que siempre 
consultan estos recursos educativos fuera del aula tradicional.

Queda por analizar los motivos por los que un grupo, aunque no muy representativo (10\% casi siempre y 3,33\% a veces) no entraron en esta categoría, considerándose que puede estar relacionado con la labor de orientación, promoción y divulgación por parte de los docentes; elementos que también fueron estudiados y se procesó en la figura 2 .

La colaboración entre estudiantes permite que unos aprendan de otros, lo cual es un elemento positivo en el proceso formativo al propiciar la socialización de lo que saben. En tal sentido, se conoció que el 86,67\% (cuarta gráfica de la figura 1) de los encuestados manifestaron que siempre comparten conocimientos con sus compañeros relacionados con el uso de las TIC y los videos relacionados con la asignatura.

Cuando en los educandos se fomenta el trabajo autónomo, prima el deseo de aprender y el interés por adquirir los conocimientos. A tales efectos, es significativo como se observa en la quinta gráfica de la figura 1, que el 93,33\% opinaron categóricamente que el modelo de aula invertida fomenta en ellos el trabajo autónomo. De igual manera, en la medida en que se eleve el interés también crecerá la motivación, por lo que no es de extrañarse que todos los encuestados (30 estudiantes que representan el $100 \%$ ) como se indica en la última gráfica de figura 1, aunaron criterios al considerar que este modelo eleva su motivación más que el de la clase tradicional.
¿El docente profundiza en el uso del recurso educativo (vídeos) en las clases? ¿El docente comparte vídeos
de histología veterinaria en
las redes sociales?
¿El docente orienta la consulta de recursos educativos disponibles en YouTube?
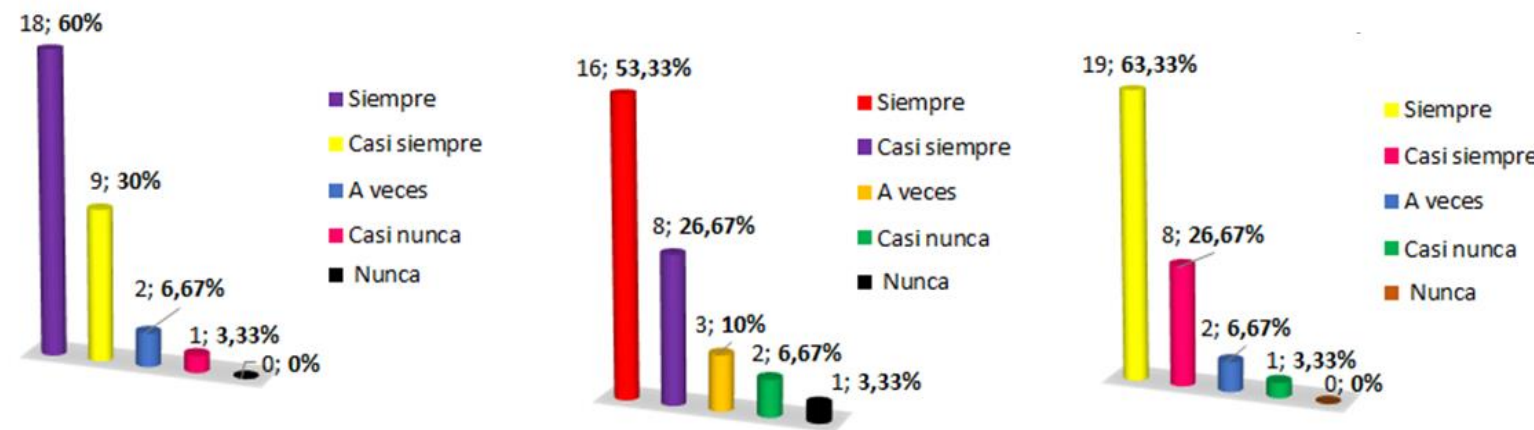

Figura 2. Resultados del procesamiento de las preguntas de la encuesta relacionadas con las acciones que realiza el docente sobre el uso y promoción de los recursos educativos.

Fue interés del estudio que se realiza, conocer también el criterio de los estudiantes sobre las acciones del docente de Histología Veterinaria en el uso y promoción de los recursos educativos. Aunque los criterios fueron variados como se puede observar en la figura 2, las opiniones a favor (siempre y casi siempre) tuvieron un alto puntaje, lo que indica que al menos se ha trabajado en este sentido.

En la primera gráfica de la figura 2 se puede apreciar que el $90 \%$ considera que siempre o casi siempre el docente profundiza el uso del recurso educativo (el video) en las clases. Si al menos este porciento de estudiantes realizó esta valoración, es porque en un número representativo de turnos de clase se está poniendo en práctica, lo que indica que el profesor reconoce sus ventajas y existe la disposición de utilizarlo. Quizás en los momentos que no se emplea sea porque no cuente con las condiciones tecnológicas para hacerlo o sean actividades prácticas donde lo educandos deban aplicar lo aprendido en teoría.

Con el auge que tienen las redes sociales en la actualidad sobre todo por adolescentes y jóvenes, las mismas deben ser vistas como una oportunidad para atraer la atención de los educandos. El compartir videos o indicar el estudio de los disponibles en ellas como en YouTube, puede además de facilitar la socialización, constituir un entorno motivador para los estudiantes y una forma efectiva de acercarse más a ellos. Como se puede apreciar en la segunda gráfica de la figura 2, el 80 $\%$ considera que siempre o casi siempre el docente comparte videos de Histología Veterinaria en las redes sociales. Respecto a la orientación de la consulta de los recursos educativos disponibles en YouTube, la última gráfica de la figura 2 indica que el $90 \%$ de los alumnos opina que se hace con cierta regularidad (siempre o casi siempre). Aunque aún se puede trabajar en beneficio del proceso educativo con mayor intencionalidad en 
este sentido, estos valores indican que ya existe un accionar en esta dirección.

\section{Discusión}

Al realizarse un análisis de los resultados obtenidos del procesamiento de la encuesta realizada a los estudiantes, se pudo apreciar criterios favorables para establecerse en la asignatura de Histología Veterinaria la clase invertida como metodología y los vídeos como instrumento de apoyo. Entre los puntos a favor puede mencionarse la disposición de los educando por este modelo, el reconocimiento que fomenta el trabajo autónomo y eleva su motivación, la colaboración entre ellos sobre los conocimientos acerca del uso de diferentes recursos educativos, la alta audiencia en la consulta de videos dentro y fuera del aula tradicional; empleando las redes sociales como YouTube para la socialización y estudio de los mismo.

Investigaciones similares respecto a la percepción de los alumnos acerca de la incorporación de la metodología de aula invertida realizado por Sánchez, Sánchez y Ruiz (2019), en la Universidad de Málaga en España y por Aguayo, Bravo, Nocetti, et al. (2019), en la Universidad del BíoBío en Chile, arrojaron opiniones en el mismo sentido dada las ventajas que reconocen en el plano didáctico que ofrece este modelo pedagógico.

Diversos estudios respaldan y promueven la implementación del aula invertida en el nivel de educación superior en el contexto ecuatoriano (Rivera y García, 2018; Pérez, Jordán y Salinas, 2018; Aycart, 2019; Cobeña y Rodríguez, 2019; Santacruz, Fernández, Martínez, et al., 2019).

Andrade, Yumi y Ramos (2020), sostiene que constituye un enfoque innovador de aprendizaje, con ventajas reales en esta era digital que rompen el modelo tradicional y mejora el desempeño del alumno en su vida académica.

En esta experiencia se coincide con Deiraola, Cambiaggi, Vita \& Zuccolilli (2018), informan de resultados favorables en la implementación del modelo de referencia en la asignatura de Osteología y Sindesmología de los miembros torácico y pelviano del canino en el corto y mediano plazo, en la carrera de Ciencias Veterinarias de la UNLP, Argentina. Los autores argumentan un alto de grado de aceptación del Aula Invertida por parte de los educandos.

También en el ámbito internacional se conoce de varias investigaciones que se han pronunciado a favor de esta práctica educativa sustentadas en el criterio que mejora el rendimiento académico, tal es el caso de Merla y Yáñez (2016); así como,
Mendaña, Poy, González, et al. (2017). En este último, tras una investigación de contraste de estudiantes de diferentes grados de una misma rama de conocimiento, en diferentes cursos y en asignaturas pudieron comprobar que se obtuvieron resultados similares en relación a la motivación y el rendimiento académico de los alumnos.

A tono con los criterios expresados por los alumnos de Histología Veterinaria, se reconoce que no son pocos los beneficios que tiene emplear el video para la comprensión de los contenidos. Se coincide con Ros y Rosa (2015), en que se eleva el interés de los estudiantes, se obtienen mayores niveles de logro, se convierten en protagonistas de su aprendizaje, se fomenta el trabajo autónomo, se incrementa su motivación y se contribuye a una adecuada gestión de su tiempo.

No son pocos las experiencias de docentes que utilizando este recurso educativo han reportado resultados satisfactorios. En este sentido puede mencionase por ejemplos en la enseñanza de la Histología Veterinaria a García, Revuela \& Lorenzo (2019), los autores argumentan el uso de metodologías innovadoras a través del Aula Invertida con el propósito de lograr un aprendizaje más dinámico y eficaz en los estudiantes.

Cabe señalar otra experiencia en la que se concuerda con el modelo flipped classroom en la enseñanza de técnicas avanzadas en laboratorios de análisis de residuos de medicamentos veterinarios y contaminantes, los investigadores indican que es modelo en la que se involucranlos alumnos en su propio aprendizaje y les permite interactuar constantemente con su entorno educativo, solucionando problemas y tomando decisiones, como otras metodologías activas a través de las TIC, Melo \& Sánchez (2017).

En concordancia con la disposición e interés del docente de Histología Veterinaria de emplear las redes sociales entre la que figura YouTube, para compartir audiovisuales e indicar la consulta de otros recursos educativos disponibles en ella, diversos estudios de especialistas en el tema avalan de positiva esta práctica. Tal es el caso de Laurencio, Pardo y Izquierdo (2018), Marín y Cabero (2019), Martínez, Valledor y Avila (2019) y Soler, Trujillo y Arias (2019). Ellos sostienen que resulta provechoso en el ámbito académico, dada su naturaleza motivadora y las facilidades de intercambio, interacción y colaboración.

Rigo, Riccetti, Siracusa, et al. (2019), muestra tres experiencia de clase invertida en la Universidad Nacional de Río Cuarto en Argentina, donde se emplean las redes sociales (en este caso Facebook) para compartir videos $y$ en algunos casos 
presentaciones en PowerPoint. Como resultado estos autores percibieron que el modelo es aplicable a diversos campos disciplinares; además, que se promovió una dinámica autónoma de trabajo y se favoreció el compromiso y aprendizaje activo los educandos.

\section{Conclusiones}

Con el estudio realizado se pudo conocer que los estudiantes y docentes tienen conocimiento del modelo del aula invertida y manifiestan que les gustaría implementarlo en la asignatura de histología veterinaria, es evidente que esto puede elevar la motivación y fortalecer la innovación pedagógica. Además, este modelo promueve el trabajo autónomo y la colaboración de los educandos en el uso de los vídeos educativos dentro y fuera del aula tradicional; empleando las redes sociales como YouTube.

\section{Bibliografía}

Aguayo, M., Bravo, M., Nocetti, A., Concha, L. \& Aburto, R. (2019). Perspectiva estudiantil del modelo pedagógico flipped classroom o aula invertida en el aprendizaje del Inglés como lengua extranjera. Revista Educación, 43(1), 97-112. Recuperado de https://doi.org/10.15517/revedu.v43i1.315 $\underline{29}$

Andrade, J. , Yumi, L. \& Ramos, R. (2020). Aula invertida de educación superior: Una revisión de literatura reciente. Revista Conciencia Digital, 3(1.2), 80-91. Recuperado de https://doi.org/10.33262/concienciadigital. v3i1.2.1180

Aycart, F. (2019). Aprendizaje invertido como un enfoque para la calidad formativa universitaria en Ecuador. Revista Conrado, 15(68), 14-21. ergmann, J. \& Sams, A. (2012).Flip your Classroom: Reach Every Student in Every Class Every day. Washington, DC: ISTE; and Alexandria, VA: ASCD.

Berenguer, C. (2016). Acerca de la utilidad del aula invertida o flipped classroom. XIV Jornadas de redes de investigación en docencia universitaria. Investigación, innovación y enseñanza universitaria: enfoques pluridisciplinares, 1466-1480. Alicante, España: Universitatd'Alacant.

Cobeña, M. Á. y Rodríguez, M. (2019). La clase invertida como modelo de investigación pedagógica. CIENCIAMATRIA. Revista
Interdisciplinaria de Humanidades, Educación, Ciencia y Tecnología, 5(1), 315.Recuperado de https://doi.org/10.35381/cm.v5i1.236

De Iraola, J., Cambiaggi, V., Vita, M. \&Zuccolilli, G. (2018). Implementación de un aula invertida para el desarrollo de los contenidos de Osteología y Sindesmología de los miembros de caninos. I Jornadas de Inclusión de Tecnologías Digitales en la Educación Veterinaria, la Plata, Argentina. Recuperado de http://sedici.unlp.edu.ar/bitstream/handle/1 0915/71763/Documento_completo.pdfPDFA.pdf? sequence $=1$ \&isAllowed $=\mathrm{y}$

García, R., Revuelta, L. \& Lorenzo, P. (2019). Aplicando metodología de aula invertida en seminarios de fisiología veterinaria. Revista Docencia Veterinaria, 3. Recuperado de http://www.vetdoc.es/index.php?journal=v etdoc\&page=article\&op=view\&path\%5B $\% 5 \mathrm{D}=2843 \&$ path\%5B $\% 5 \mathrm{D}=2815$

Kachka, P. (2012). Understanding the flipped classroom: Part 1.Recuperado de https://www.facultyfocus.com/articles/blen ded-flipped-learning/understanding-theflipped-classroom-part-1/

Laurencio, K., Pardo, M. E. \&Izquierdo, J. M. (2018). Reflexión acerca del empleo de las redes sociales, con fines educativos, en la Educación Superior. Revista Opuntia Brava,10(3), 263-273. https://doi.org/10.35195/ob.v10i3.557

Marín, V. \& Cabero, J. (2019). Las redes sociales en educación: Desde la innovación a la investigación educativa. RIED. Revista Iberoamericana de Educación a Distancia, 22(2).

https://doi.org/10.5944/ried.22.2.24248

Martínez, L., Valledor, R. F. \& Avila, Y. de la C. (2019). Las redes sociales de internet para la gestión del conocimiento en el contexto de la Educación Superior. Revista Tecnología Educativa, 4(1), 25-31. https://tecedu.uho.edu.cu/index.php/tecedu /article/view/107/89

Melo, L. \& Sánchez, R. (2017).Análisis de las percepciones de los alumnos sobre la metodología flipped classroom para la enseñanza de técnicas avanzadas en laboratorios de análisis de residuos de medicamentos veterinarios $y$ 
contaminantes. Revista Educación Química, 28,(1), 30-37. Recuperado de https://doi.org/10.1016/j.eq.2016.09.010

Mendaña, C., Poy, R., González, A., Arana, M. V. \& López, E. (2017). ¿influye el aula invertida en la motivación y el rendimiento académico de estudiantes universitarios? Revista Infancia, Educación y Aprendizaje, 3(2), 660-666. Recuperado dehttps://doi.org/10.22370/ieya.2017.3.2. $\underline{798}$

Merla, A. \& Yáñez, C. (2016). El aula invertida como estrategia para la mejora del rendimiento académico. Revista Mexicana de Bachillerato a Distancia, 8(16), 68. https://doi.org/10.22201/cuaed.20074751e. $\underline{2016.16 .57108}$

Monteagudo, J., Gómez, C. \& Miralles, P. (2017). Evaluación del diseño e implementación de la metodología. Revista de Educación a Distancia. 55, (7), 22- 12.Recuperado de http://dx.doi.org/10.6018/red/55/7

Pérez, V. Jordán, E. \& Salinas, L. (2018). Didáctica del aula invertida y la enseñanza de física en la Universidad Técnica de Ambato. Mikarimin. Revista Científica Multidisciplinaria, 4(3), 111-126. Recuperado de http://45.238.216.13/ojs/index.php/mikari $\underline{\mathrm{min} / \mathrm{article} / \mathrm{view} / 1340}$

Rigo, D. Y., Riccetti, A. E., Siracusa, M. \& Paolini, P. (2019). Tres experiencias sobre clases invertidas para promover el compromiso por el aprendizaje. Percepciones de estudiantes universitarios. Páginas de Educación, 12(2), 43-58. https://doi.org/10.22235/pe.v12i2.1836

Rivera, M. \& García, A.(2018).Aula invertida con tecnologías emergentes en ambientes virtuales en la Universidad Politécnica Salesiana del Ecuador. Revista Cubana de Educación Superior, 37 (1). Recuperado dehttp://scielo.sld.cu/pdf/rces/v37n1/rces0 8118.pdf

Ros, A. \& Rosa, A. (2015). Uso del vídeo docente para la clase invertida: Evaluación, ventajas e inconvenientes. En Vectores de la pedagogía docente actual (pp. 423-441). ACCI (Asociación Cultural y Científica Iberoamericana). Recuperado de https://www.researchgate.net/profile/Alfon so_RosaGarcia/publication/266673438_Us o_del_video_docente_para la clase invert ida evaluacion_ventajas e inconvenientes /links/570c9bb608aea660813b2c87/Usodel-video-docente-para-la-clase-invertidaevaluacion-ventajas-e-inconvenientes.pdf

Sánchez, N., Sánchez, T. \& Pérez, O. (2015). El video en la enseñanza de la Física: Un recurso didáctico contemporáneo. Revista Magisterio, 27. Recuperado de https://revista.uij.edu.cu/index.php/magiste $\underline{\text { rio/article/view/304/268 }}$

Sánchez, E., Sánchez, J. \& Ruiz, J. (2019). Percepción del alumnado universitario respecto al modelo pedagógico de clase invertida. Magis, Revista Internacional de Investigación en Educación, 11(23), 151$168 . \quad$ Recuperado de https://doi.org/10.11144/Javeriana.m1123.paur

Santacruz, A., Fernández, G. E., Martínez, C. \& Santacruz, D. (2019). Didáctica del aula invertida y la investigación formativa en la Universidad Técnica de Quevedo. Mikarimin. Revista Científica Multidisciplinaria, 5(3), 121-132. Recuperado de http://45.238.216.13/ojs/index.php/mikari $\underline{\mathrm{min} / \mathrm{article} / \mathrm{view} / 1279}$

Soler, Y., Trujillo, C. \& Arias, M. (2019). Redes sociales en la juventud, formación a través del enfoque por competencias y el aula invertida. Opuntia Brava, 11(3), 80-88. Recuperado dehttps://doi.org/10.35195/ob.v11i3.791 